diversity in the human intestine. Cell. 124:837-848. 5. Rakoff-Nahoum, S., Paglino, J., Eslami-Varzaneh, F., Edberg, S., and Medzhitov, R. 2004. Recognition of commensal microflora by toll-like receptors is required for intestinal homeostasis. Cell. 118:229-241

6. Kitajima, S., Morimoto, M., Sagara, E., Shimizu, C., and Ikeda, Y. 2001. Dextran sodium sulfateinduced colitis in germ-free IQI/Jic mice. Exp. Anim. 50:387-395

7. Pull, S.L., Doherty, J.M., Mills, J.C., Gordon, J.I., and Stappenbeck, T.S. 2005. Activated macrophages are an adaptive element of the colonic epithelial progenitor niche necessary for regenerative responses to injury. Proc. Natl. Acad. Sci. U. S. A. 102:99-104.

8. Takeda, K., Kaisho, T., and Akira, S. 2003. Toll-like receptors. Annu. Rev. Immunol. 21:335-376.

9. Araki, A., et al. 2005. MyD88-deficient mice develop severe intestinal inflammation in dextran sodium sulfate colitis. J. Gastroenterol. 40:16-23.

10. Fukata, M., et al. 2005. Toll-like receptor-4 is required for intestinal response to epithelial injury and limiting bacterial translocation in a murine model of acute colitis. Am. J. Physiol. Gastrointest. Liver Physiol. 288:G1055-G1065.

11. Rakoff-Nahoum, S., Hao, L., and Medzhitov, R. 2006. Role of toll-like receptors in spontaneous commensaldependent colitis. Immunity. 25:319-329.

12. Booth, D., and Potten, C.S. 2001. Protection against mucosal injury by growth factors and cytokines. J. Natl. Cancer Inst. Monogr. 2001:16-20.

13. Morteau, O., et al. 2000. Impaired mucosal defense to acute colonic injury in mice lacking cyclooxygenase-1 or cyclooxygenase-2.J. Clin. Invest. 105:469-478.

14. Tessner, T.G., Cohn, S.M., Schloemann, S., and Stenson, W.F. 1998. Prostaglandins prevent decreased epithelial cell proliferation associated with dextran sodium sulfate injury in mice. Gastroenterology. 115:874-882.

15. Rhee, S.H., and Hwang, D. 2000. Murine TOLLlike receptor 4 confers lipopolysaccharide responsiveness as determined by activation of NF kappa $\mathrm{B}$ and expression of the inducible cyclooxygenase. J. Biol. Chem. 275:34035-34040.

16. Eliopoulos, A.G., Dumitru, C.D., Wang, C.C., Cho,
J., and Tsichlis, P.N. 2002. Induction of COX-2 by LPS in macrophages is regulated by Tpl2-dependent CREB activation signals. EMBO J. 21:4831-4840.

17. Houchen, C.W., Sturmoski, M.A., Anant, S., Breyer, R.M., and Stenson, W.F. 2003. Prosurvival and antiapoptotic effects of PGE2 in radiation injury are mediated by EP2 receptor in intestine. Am. J. Physiol. Gastrointest. Liver Physiol. 284:G490-G498.

18. Bjerknes, M., and Cheng, H. 2001. Modulation of specific intestinal epithelial progenitors by enteric neurons. Proc. Natl. Acad. Sci. U. S. A. 98:12497-12502.

19. Wang, D., et al. 2006. CXCL1 induced by prostaglandin E2 promotes angiogenesis in colorectal cancer. J. Exp. Med. 203:941-951.

20. Fukata, M., et al. 2006. Cox-2 is regulated by Tolllike receptor-4 (TLR4) signaling: role in proliferation and apoptosis in the intestine. Gastroenterology. 131:862-877.

21. Houchen, C.W., Stenson, W.F., and Cohn, S.M. 2000. Disruption of cyclooxygenase-1 gene results in an impaired response to radiation injury. Am. J. Physiol. Gastrointest. Liver Physiol. 279:G858-G865.

\title{
Beta blocker specificity: a building block toward personalized medicine
}

\author{
Brent R. DeGeorge Jr. and Walter J. Koch \\ Center for Translational Medicine and George Zallie and Family Laboratory for Cardiovascular Gene Therapy, \\ Department of Medicine, Thomas Jefferson University, Philadelphia, Pennsylvania, USA.
}

\begin{abstract}
Drugs known as beta blockers, which antagonize the $\beta$-adrenergic receptor $(\beta-A R)$, are an important component of the treatment regimen for chronic heart failure (HF). However, a significant body of evidence indicates that genetic heterogeneity at the level of the $\beta_{1}$-AR may be a factor in explaining the variable responses of HF patients to beta blockade. In this issue of the JCI, Rochais et al. describe how a single amino acid change in $\beta_{1}$-AR alters its structural conformation and improves its functional response to carvedilol, a beta blocker currently used in the treatment of HF (see the related article beginning on page 229). This may explain why some HF patients have better responses not only to carvedilol but to certain other beta blockers as well. The data greatly enhance our mechanistic understanding of myocardial adrenergic signaling and support the development of "tailored" or "personalized" medicine, in which specific therapies could be prescribed based on a patient's genotype.
\end{abstract}

Chronic heart failure (HF) poses a major public health problem in this country, primarily due to the increasing proportion of our society surviving to the seventh and eighth decades of life and to improved treatment of acute ischemic cardiac events, resulting in more survi-

Nonstandard abbreviations used: AC, adenylyl cyclase; $\beta$-AR, $\beta$-adrenergic receptor; FRET, fluorescence resonance energy transfer; GPCR, G protein-coupled receptor; $\mathrm{HF}$, heart failure.

Conflict of interest: The authors have declared that no conflict of interest exists.

Citation for this article: J. Clin. Invest. 117:86-89 (2007). doi:10.1172/JCI30476. vors who develop cardiac dysfunction. The group of drugs known as beta blockers block the effects of catecholamines, such as epinephrine and norepinephrine, on the body's $\beta$-adrenergic receptors ( $\beta$-ARs), slowing nerve impulses traveling through the heart and reducing the heart's workload. While $\beta$-AR antagonists have become a mainstay of HF therapy, the most recent guidelines from the American College of Cardiology and the American Heart Association acknowledge that there are unresolved issues concerning the use of these drugs for the treatment of HF (1).

\section{$\beta_{1}$-AR polymorphisms and cardiac phenotype}

The $\beta$-ARs are members of the $G$ proteincoupled receptor (GPCR) superfamily, which consists of over 700 genes that are the targets of more than $50 \%$ of the drugs in clinical practice (2). There are 3 known types of $\beta$-ARs, $\beta_{1}, \beta_{2}$, and $\beta_{3}$. The $\beta_{1}$-ARs are located mainly in the heart, kidney, and adipose tissue. The $\beta_{2}$-ARs are located mainly in the heart, lung, gastrointestinal tract, liver, pancreas, and skeletal muscle. The role and location of $\beta_{3}$-ARs are less well defined. When stimulated by agonists, $\beta$-ARs primarily activate heterotrimeric guanine nucleotide-binding $(G)$ proteins of the Gs family, causing dissociation of G $\alpha-G T P$ and $\mathrm{G} \beta \gamma$ subunits. The $\mathrm{G}$ proteins transduce intracellular signaling pathways via adenylyl cyclase (AC) activation, and this results in increased intracellular cAMP levels (3). This signaling cascade ultimately leads to positive regulatory input to the myocardial contractile apparatus. Importantly, $\beta$-ARs present on cardiomyocytes represent the most powerful means of enhancing the contractile performance of the heart.

The predominant $\beta$-AR subtypes of functional consequence to cardiac physiology are $\beta_{1}-\mathrm{AR}$ and $\beta_{2}-\mathrm{AR}$, both of which have 


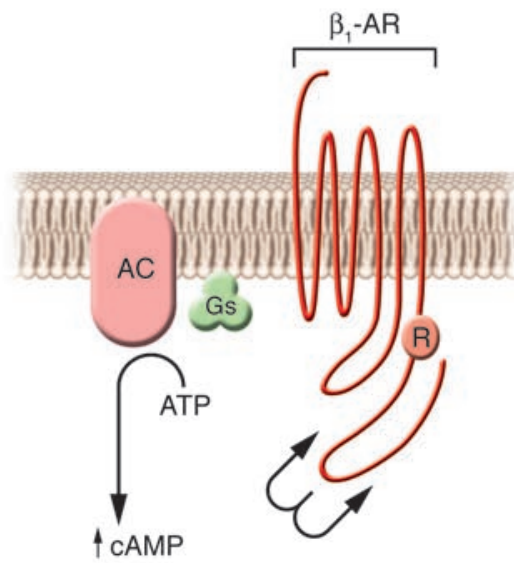

$\operatorname{Arg} 389 \beta_{1}-A R$
Agonist

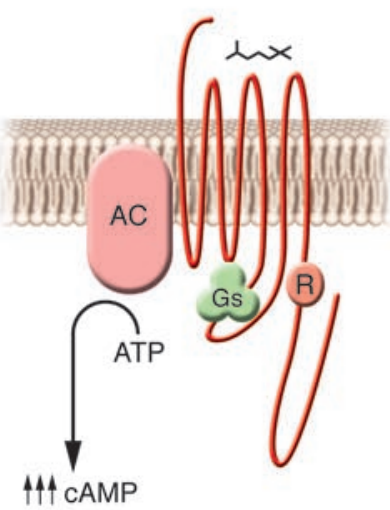

$\uparrow \uparrow \uparrow$ CAMP

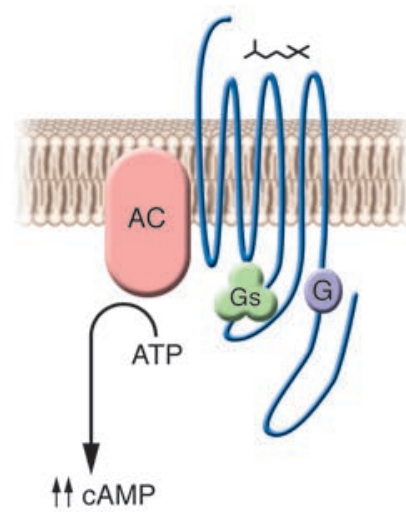

Carvedilol
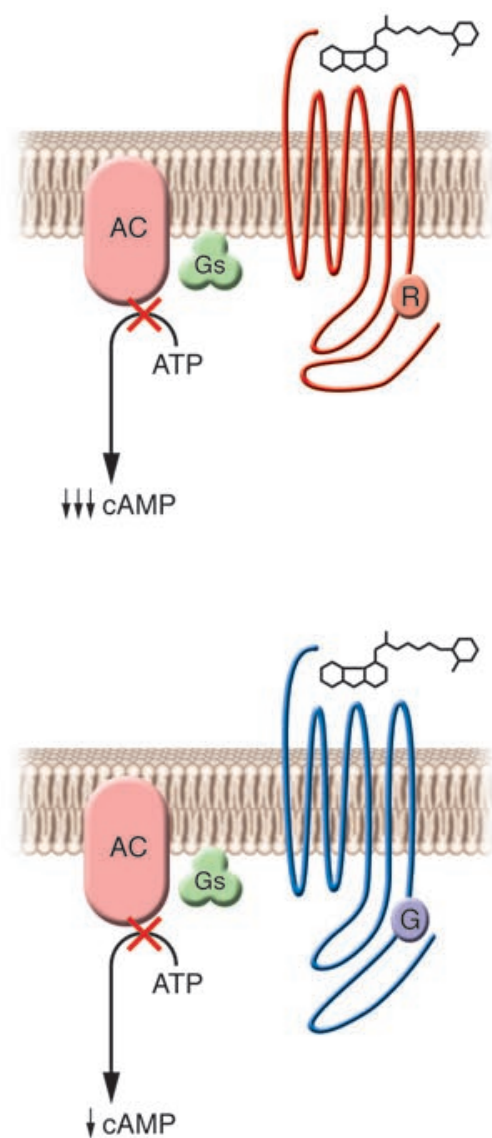

Figure 1

The effect of the $\beta_{1}$-AR Gly389Arg polymorphism on signal transduction and beta blockade by carvedilol. The Gly389Arg polymorphism of the $\beta_{1}$-AR occurs in the region between the seventh transmembrane domain and the intracellular tail of the receptor (R and $G$ correspond to arginine and glycine, respectively, at position 389 of the receptor). This highly conserved region is putatively associated with coupling to the Gs protein. The change of the amino acid residue at position 389 of the $\beta_{1}$-AR from the polar, basic arginine (upper 3 panels) to the small, nonpolar glycine (lower 3 panels) may result in a modified structure that could alter receptor-Gs interaction. This region of the cytoplasmic tail of the receptor may participate in regulating the affinity of the receptor-Gs interaction. This could potentially explain the differences in receptor-Gs coupling and cAMP production associated with the Arg389 $\beta_{1}$-AR variant as compared with the Gly389 $\beta_{1}$-AR variant reported previously $(7,8)$. Under basal conditions, the Arg389 variant has been associated with increased cAMP production, which is markedly augmented as compared with the Gly389 variant following agonist stimulation (7). This effect could also be attributed to a more favorable structural environment for interaction with Gs. Carvedilol treatment induces a conformational change resulting in the intracellular tail of the receptor being positioned more closely to the third intracellular loop; this inverse agonism of the receptor in theory would lead to decreased Gs coupling and reduced cAMP accumulation. Both variants demonstrated inverse agonist properties in response to carvedilol; however, the effect was increased 2.5-fold in the Arg389 variant, apparently due to conformation of the Arg389 structure in the presence of the ligand as observed by Rochais et al. in this issue of the $\mathrm{JCl}$ (14).

well-documented polymorphisms that exist in human populations. The coding region of the gene encoding the $\beta_{1}$-AR, located on chromosome 10q24-26, contains 2 known SNPs resulting in amino acid substitutions. At position 49 in the extracellular amino terminus of the receptor, a serine is substituted by a glycine (Ser49Gly) with an allele frequency of 0.87 and 0.13 , respectively (4). At position 389 in the intracellular carboxy terminus in the proximity of the seventh transmembrane spanning segment, a glycine or arginine can be found at an allele frequency of 0.25 and 0.75 , respectively (4). These polymorphic variants of the $\beta_{1}$-AR appear to have significant cardiovascular phenotypic consequences. For example, Ranade et al. found a significant functional association between the Ser49Gly polymorphism and resting heart rate, in which Gly49 homozygotes had the lowest heart rate, and each
Ser49 allele increased the basal heart rate in an additive model (5). The Arg389 variant appears to be linked with HF in both clinical outcomes and response to therapy (6).

More specifically, the Arg389Gly polymorphism lies within the putative Gs-binding domain of the $\beta_{1}$-AR (Figure 1), and consistent with this localization, previous in vitro studies in fibroblast cell lines transiently transfected with the Arg389 $\beta_{1}$-AR have demonstrated enhanced receptor-Gs coupling as 
measured by ${ }^{35}$ S-GTP binding and slightly increased basal and markedly increased agonist-induced AC activity as compared with Gly389 $\beta_{1}$-AR-transfected fibroblasts (7). Furthermore, in a study of transgenic mice with cardiac-specific overexpression of Arg389Gly $\beta_{1}$-AR variants, Akhter and colleagues demonstrated that not only does Arg389 $\beta_{1}$-AR lead to increased myocardial signaling properties, but it also confers cardioprotection following myocardial ischemia and reperfusion injury (8). This may involve the upregulation of GPCR kinase 2 (GRK2) activity, which was presumably induced by the increased signaling of the Arg389 variant, since the less active Gly389 form did not alter myocardial GRK2 levels.

In $\mathrm{HF}$, the myocardium has a significant loss of contractile function. In order to compensate for the loss of systemic perfusion due to pump failure, the heart must increase heart rate and contractility. This is done by increased neurohumoral activation through increases in the activity of the sympathetic nervous system and renin-angiotensin system. Increases in the sympathetic catecholamines norepinephrine and epinephrine lead to chronic activation of myocardial $\beta$-ARs in order to drive the failing heart. However, chronic activation of these pathways, in particular activation of the $\beta_{1}$-AR, leads to increased cardiotoxicity and cardiac pathology (3). This partially explains the clinical benefit achieved by $\beta$-AR antagonists, which block the overt noxious effects of catecholamines and have been shown to improve survival and reverse pathologic cardiac remodeling (9). Interestingly, sensitivity to the beneficial effects of $\beta$-AR blockade differs by ethnicity, as evidenced by data from the Beta-Blocker Evaluation of Survival Trial (BEST) demonstrating a lack of benefit in black patients with New York Heart Association class III or IV HF treated with the beta blocker bucindolol, compared with other patients (10). It was reasoned that racial differences in the incidence of HF and therapeutic response to $\beta$-AR antagonists may be heritable and that genetic heterogeneity with respect to $\beta_{1}$-ARs may partially account for this phenomenon. However, conflicting data from clinical studies analyzing the association of $\beta_{1}$-AR polymorphisms and response to $\beta$-AR antagonists have made it difficult to reach conclusions regarding the true clinical importance of $\beta$-AR polymorphisms with respect to treatment outcome (for review see ref. 11).

\section{$\beta_{1}$-AR signaling in real time}

Recent advances in the fields of biochemistry and applied science have generated new classes of fluorescent probes to permit the direct assessment of the dynamic behavior of biological molecules. Specifically, fluorescence resonance energy transfer-based (FRETbased) techniques have allowed investigators to evaluate dynamic protein-protein interactions and protein conformational changes in real time (for reviews see refs. 12, 13). FRET fundamentally characterizes the spatiotemporal relationship of fluorescently labeled molecules within a system by way of recording the energy transfer from a donor fluorophore in an excited electronic state to an acceptor fluorophore of adequate proximity. In this issue of the JCI, Rochais and colleagues report on their exploitation of FRET technology to generate a $\beta_{1}$-AR FRET sensor to directly assess the effects of the Arg389Gly polymorphism in the $\beta_{1}$-AR on receptor activation, downstream signal transduction, and response to beta blockade in cultured human cells in real time (14). The $\beta_{1}$-AR FRET sensor used in this study was generated based on evidence that the receptor undergoes conformational changes upon agonist binding, specifically in the region of the carboxy terminus and third intracellular loop, which move apart upon receptor activation. The authors then tagged these regions with cerulean and yellow fluorescent proteins, respectively, allowing them to detect the movement of these regions in real time using FRET, thereby tracking the dynamic changes in the receptor in response to agonist and antagonist binding. The authors demonstrated that this $\beta_{1}$-AR FRET sensor shares pharmacological and signaling characteristics consistent with that of the WT $\beta_{1}$-AR, including similar activation kinetics and downstream signaling via cAMP formation.

Utilizing $\beta_{1}$-AR FRET sensors for both Gly389 and Arg389 variants of the $\beta_{1}$-AR, the authors were able to directly assess the functional consequences of $\beta$-AR polymorphisms with respect to receptor conformation in response to pharmacologic agonists and antagonists. In contrast with results in previous in vitro studies $(7,8)$, the authors identified no significant differences between Gly389 and Arg389 variants in $\beta_{1}$-AR-mediated Gs coupling or cAMP accumulation in response to agonist (14).

Perhaps the most exciting clinically relevant data from this study is the comparison of the FRET responses of the $2 \beta_{1}$-AR variants generated by the $\beta$-AR antagonists bisoprolol, metoprolol, and carvedilol.
Each $\beta$-AR antagonist induced an increase in FRET ratio, signifying the occurrence of an active change in receptor conformation upon antagonist binding, suggesting an inverse agonist mechanism of action on the $\beta$-AR. Increases in FRET ratio were minor with respect to decreases in receptor activation for metoprolol and bisoprolol, and the effects of these 2 beta blockers did not differ between $\beta_{1}$-AR variants (14). In contrast, carvedilol induced strong inverse agonism with regard to both $\beta_{1}$-AR variants; furthermore, the Arg389 $\beta_{1}$-AR variant exhibited a 2.5-fold increase in FRET ratio as compared with the Gly389 $\beta_{1}$-AR variant. The unique properties of this beta blocker were confirmed downstream of receptor inactivation at the level of decreased cAMP accumulation: both $\beta_{1}$-AR variants demonstrated marked reduction in basal cAMP levels (14). Carvedilol led to a much stronger reduction in basal cAMP content in the Arg389 $\beta_{1}$-AR variant as compared with the Gly389 $\beta_{1}$-AR variant (see Figure 1). The authors further established the phenotypic relevance of the molecular alterations induced by the Arg389Gly polymorphisms using primary neonatal rat cardiac myocytes infected with adenoviruses containing the $\beta_{1}$-AR variants as a model system to study cardiac rate control. Under basal conditions, both $\beta_{1}$-AR variants induced an increase in contractile activity as compared with WT cardiac myocytes; however, the contractile activity of the Arg389 variant was increased nearly 1.5 -fold as compared with the Gly389 variant, and carvedilol significantly decreased this activity preferentially in the Arg389 $\beta_{1}$-AR.

Overall, the study by Rochais et al. (14) represents the first direct assessment of GPCR function using the well-characterized $\beta_{1}$-AR system that also happens to be critical for normal and pathological cardiac physiology. Of importance, the authors were able to assess the conformational changes that occurred during agonism and antagonism of the receptor. Moreover, the data reveal that different $\beta_{1}$-AR antagonists, particularly carvedilol, are capable of inducing varying degrees of inverse agonism on the receptor and that this effect of carvedilol is dependent on the amino acid residue present at position 389 of the $\beta_{1}$-AR. Carvedilol, unlike metoprolol or bisoprolol, is a nonselective beta blocker and can also act on $\alpha_{1}$-ARs (15). Therefore, it would be interesting to address the potential effects of $\beta_{2}$-AR and $\alpha_{1}$-AR signaling on cardiac rate control to ascertain whether any receptor crosstalk or dimerization is responsible for the decreased basal 
cAMP production mediated by the Arg389 variant or whether antagonism of these other receptors is involved in the beneficial effects of carvedilol in HF. However, what is important to remember based on the current work is that for the $\beta_{1}$-AR, carvedilol displays the greatest degree of inverse agonism, especially for the Arg389 variant (Figure 1), and studies such as this could lead to research broadening our knowledge of this pharmacological property of GPCR ligands and therefore potentially increase their clinical utility (16).

\section{Taking it personally}

The implications of these findings are of potentially profound clinical importance when considering the interindividual and ethnic variation that occurs in response to $\beta$-AR blocker therapy in the treatment of HF. It has been reported that the allele frequency of the Arg389 variant is $20 \%$ less common in black patients compared with non-black patients, and this may partially explain the poorer response to $\beta_{1}$-AR antagonists seen in blacks compared with that of the rest of the population (17). Future efforts to characterize the effect of genetic heterogeneity in cellular response to pharmacological agents, such as the $\beta_{1}$-AR antagonists examined in this study, lend credence to the viability of the concept of pharmacogenomics - pharmacologic intervention adapted according to an individual's genetic makeup - and the realization of "personalized" medicine for the treatment of cardiovascular disorders such as HF (18).

Address correspondence to: Walter J. Koch, Center for Translational Medicine, George Zallie and Family Laboratory for Cardiovascular Gene Therapy, Department of Medicine, Thomas Jefferson University, 1025 Walnut Street, Room 317, Philadelphia, Pennsylvania 19107, USA. Phone: (215) 955-9982; Fax: (215) 503-5731; E-mail: walter.koch@jefferson.edu.

1. Lee, T.H. 2001. Management of heart failure: AHA/ ACC guidelines summary. Heart disease: a textbook of cardiovascular medicine. 6th edition. E. Braunwald, editor. 101:652-685.

2. Becker, O.M., et al. 2004. G-protein coupled receptors: in silico drug discovery in 3D. Proc. Natl. Acad. Sci. U. S. A. 101:11304-11309.

3. Rockman, H.A., Koch, W.J., and Lefkowitz, R.J. 2002. Seven-transmembrane-spanning receptors and heart function. Nature 415:206-212.

4. Maqbool, A., Hall, S.A., Ball, S.G., and Balmforth, A.J. 1999. Common polymorphisms of $\beta_{1}$-adrenoreceptor: identification and rapid screening assay [research letter]. Lancet. 353:897.

5. Ranade, K., et al. 2002. A polymorphism in the $\beta_{1}$ adrenergic receptor is associated with resting heart rate. Am. J. Hum. Genet. 70:935-942.

6. Small, K.M., McGraw, D.W., and Liggett, S.B. 2003 Pharmacology and physiology of human adrenergic receptor polymorphisms. Annu. Rev. Pharmacol. Toxicol. 43:381-411

7. Mason, D.A., Moore, J.D., Green, S.A., and Liggett, S.B. 1999. A gain-of-function polymorphism in a Gprotein coupling domain of the human $\beta_{1}$-adrenergic receptor. J. Biol. Chem. 274:12670-12674.
8. Akhter, S.A., D’Souza, K.M., Petrashevskaya, N.N., Mialet-Perez, J., and Liggett, S.B. 2006. Myocardial $\beta_{1}$-adrenergic receptor polymorphisms affect functional recovery after ischemic injury. Am. J. Physiol. Heart Circ. Physiol. 290: H1427-H1432.

9. Brauwald, E., and Bristow, M.R. 2002. Congestive heart failure: fifty years of progress. Circulation. 102(20 Suppl. 4):IV14-IV23.

10. Beta-blocker Evaluation of Survival Trial Investigators. 2001. A trial of the beta-blocker bucindolol in patients with advanced chronic heart failure. N. Engl. J. Med. 344:1659-1667.

11. Leinweber, K. 2004. Beta-adrenergic receptor polymorphism in human cardiovascular disease. Ann. Med. 36:64-69.

12. Giempmans, B.N., Adams, S.R., Ellisman, M.H., and Tsien, R.Y. 2006. The fluorescent toolbox for assessing protein localization and function. Science. 312:217-224.

13. Miyawaki, A. 2003. Visualization of the spatial and temporal dynamics of intracellular signaling. Dev. Cell. 4:295-305.

14. Rochais, F., et al. 2007. Real-time optical recording of $\beta_{1}$-adrenergic receptor activation reveals supersensitivity of the Arg389 variant to carvedilol. J. Clin. Invest. 117:229-235. doi:10.1172/JCI30012.

15. Yancy, C.W., et al. 2001. Race and the response to adrenergic blockade with carvedilol in patients with chronic heart failure. N. Engl. J. Med. 344:1358-1364.

16. Bond, R.A., and Ijzerman, A.P. 2006. Recent developments in constitutive receptor activity and inverse agonism, and their potential for GPCR drug discovery. Trends Pharmacol. Sci. 27:92-96.

17. Small, K.M., Wagoner, L.E., Levin, A.M., Kardia, S.L., and Liggett, S.B. 2002. Synergistic polymorphisms of $\beta_{1^{-}}$and $\alpha_{2 \mathrm{c}}$-adrenergic receptors and the risk of congestive heart failure. N. Engl. J. Med. 347:1135-1142.

18. Zineh, I., and Johnson, J.A. 2006. Pharmacogenetics of chronic cardiovascular drugs: applications and implications. Expert Opin. Pharmacother. 11:1417-1427.

\title{
Macrophage heterogeneity and tissue lipids
}

\author{
Siamon Gordon
}

Sir William Dunn School of Pathology, University of Oxford, Oxford, United Kingdom.

\begin{abstract}
Macrophages are present as resident cells in adipose tissue, and blood monocytes are recruited in increased numbers to sites of lipid accumulation in atherosclerosis, a modified form of inflammation in the arterial wall. Recent findings reported by 3 separate groups in this issue of the JCI provide evidence for distinct monocyte subsets, differential chemokine receptor usage, and phenotypic modulation of macrophages in murine models of genetic and high-fat diet-induced disease (see the related articles beginning on pages 175, 185, and 195). These studies raise prospects for selective therapeutic targets to ameliorate macrophage hyperinflammatory responses, while sparing host defense and repair mechanisms.
\end{abstract}

Nonstandard abbreviations used: CCR2, C-C motif chemokine receptor 2; CX3CR1, C- $\mathrm{X}_{3}-\mathrm{C}$ motif chemokine receptor 1 .

Conflict of interest: The author has declared that no conflict of interest exists.

Citation for this article: J. Clin. Invest. 117:89-93 (2007). doi:10.1172/JCI30992.
Monocytes and mature macrophages are prominent in the host response to lipid accumulation in major arteries, the development of atherosclerotic plaques, and their complications (1). Less established are the details of their colocalization and possible metabolic interactions with adi- pocytes in body fat stores (2). New studies reported in this issue of the JCI by Swirski et al. (3) demonstrate that a monocyte subset that expresses high levels of a marker antigen, Ly-6C, dominates hypercholesterolemia-associated monocytosis and gives rise to macrophages in atheromata. Also in this issue, Tacke et al. (4) report that monocyte subsets differentially employ the chemokine receptors $\mathrm{C}-\mathrm{C}$ motif chemokine receptor 2 (CCR2), CCR5, and C- $\mathrm{X}_{3}-\mathrm{C}$ motif chemokine receptor 1 (CX3CR1, also known as the fractalkine receptor) to enter atherosclerotic plaques. They also exploited an mAb, Gr-1, directed against Ly- 6 family antigens to distinguish monocyte subsets and used CD11c, a $\beta 2$ integrin expressed by myeloid DCs and selected tis- 